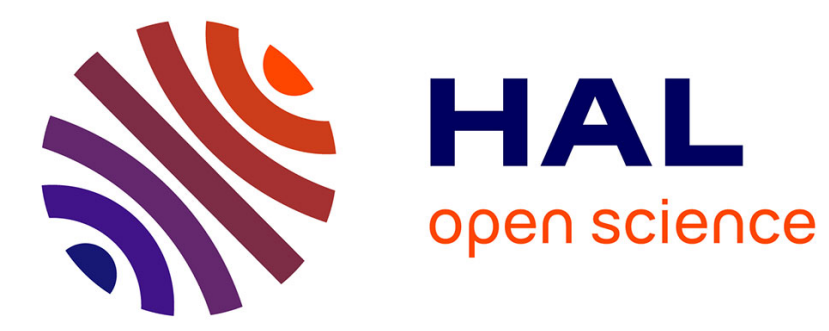

\title{
Optical Slot Switching Latency in Mobile Backhaul Networks
}

Nihel Benzaoui, Yvan Pointurier, Thomas Bonald, Qing Wei, Matthias Lott

\section{To cite this version:}

Nihel Benzaoui, Yvan Pointurier, Thomas Bonald, Qing Wei, Matthias Lott. Optical Slot Switching

Latency in Mobile Backhaul Networks. ECOC, 2014, Cannes, France. hal-01244331

\section{HAL Id: hal-01244331 \\ https://inria.hal.science/hal-01244331}

Submitted on 15 Dec 2015

HAL is a multi-disciplinary open access archive for the deposit and dissemination of scientific research documents, whether they are published or not. The documents may come from teaching and research institutions in France or abroad, or from public or private research centers.
L'archive ouverte pluridisciplinaire HAL, est destinée au dépôt et à la diffusion de documents scientifiques de niveau recherche, publiés ou non, émanant des établissements d'enseignement et de recherche français ou étrangers, des laboratoires publics ou privés. 


\title{
Optical Slot Switching Latency in Mobile Backhaul Networks
}

\author{
N. Benzaoui ${ }^{(1)}$, Y. Pointurier ${ }^{(1)}$, T. Bonald ${ }^{(2)}, \mathrm{Q} . \mathrm{Wei}^{(3)}, \mathrm{M}$. Lott ${ }^{(3)}$ \\ (1) Alcatel-Lucent Bell Labs, Nozay, France, Nihel Dioher.Benzaoui@alcatel-lucent.com \\ (2) Telecom ParisTech, Paris, France; and LINCS, Paris, France \\ (3) DOCOMO Communications Laboratories Europe $\mathrm{GmbH}$, Munich, Germany
}

\begin{abstract}
We show that an optical slot switching network fulfills the strict latency constraints of a next generation LTE advanced switching mobile backhaul network. The impact of class-of-service handling when aggregating client data into slots is also quantified.
\end{abstract}

\section{Introduction}

With the evolution of the optical transport technologies, energy-efficient solutions for the transport in the radio access part of cellular backhaul networks have become available. In this paper, we consider the use of fine granularity optical transport solutions - optical slot switching (OSS), an energy-efficient switching technology ${ }^{1}$, to interconnect the eNodeBs in an LTE mobile backhaul network. Here, optical slot switching is applied at the metro level for backhaul networks with a few (and up to a few dozens) of nodes (eNodeBs): Fig. 1 shows LTE base-stations (eNodeBs) interconnected by a mobile backhaul network.

We focus on Packet Optical Add/Drop Multiplexer (POADM ${ }^{3}$; see top of Fig. 1), an implementation of OSS for physical ring networks. POADM provides sub-wavelength switching granularity, and is designed to minimize the power consumption thanks to optical transparency for the transit traffic ${ }^{1}$. Direct node-to-node communication ${ }^{2}$ can be efficiently leveraged to transport Coordinated MultiPoint (CoMP) traffic, which is used in the LTE standards to improve coverage, cell-edge throughput, and system efficiency.

This paper quantifies for the first time the latency of an OSS-based LTE advanced backhaul network using real traffic demand. Specifically, we show that the overall latency is compatible with LTE advanced requirements. In addition, we investigate the impact of class of service (CoS) handling at the slot level to support additional real-time traffic, at the expense of a reduction of the maximum amount of non-real-time traffic that can be carried before losses occur.

\section{Support of differentiated classes of service}

Traffic in an LTE advanced mobile backhaul network is described in Tab. 1. In this work we consider 2 classes of service: real-time (RT) and non-real-time (NRT). RT traffic is latency sensitive and consists of (some) data traffic, Voice over IP (VoIP), and signaling for CoMP,

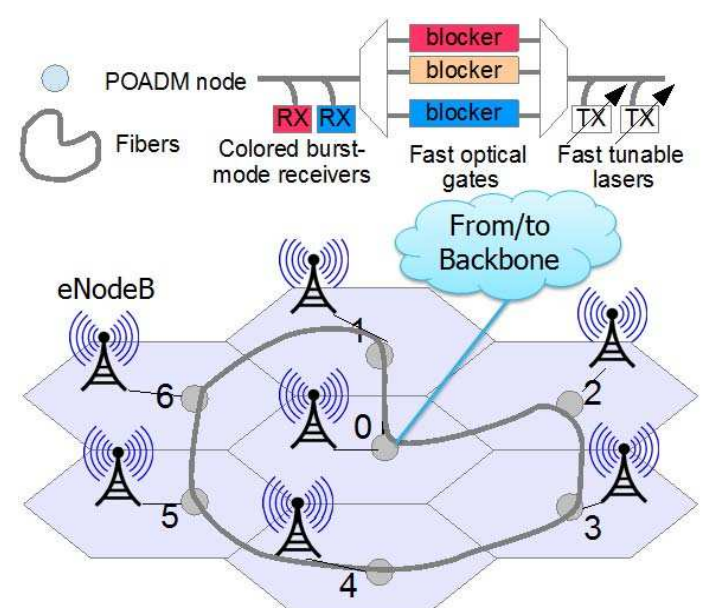

Figure 1: POADM mobile backhaul ring.

Table 1: Traffic characteristics. ${ }^{1,8}$

\begin{tabular}{|l|l|l|l|}
\hline Type & Demand & Pattern & CoS \\
\hline Data & $\begin{array}{l}1.5 \mathrm{~Gb} / \mathrm{s} \text { up, } \\
3 \mathrm{~Gb} / \mathrm{s} \text { down }\end{array}$ & Centralized & $\begin{array}{l}\text { RT, } \\
\text { NRT }\end{array}$ \\
\hline $\begin{array}{l}\text { Voice } \\
\text { over IP }\end{array}$ & $\begin{array}{l}8.5 \mathrm{Mb} / \mathrm{s} \text { up } \\
\text { and down }\end{array}$ & Centralized & RT \\
\hline $\begin{array}{l}\text { CoMP } \\
\text { data }\end{array}$ & $1 \mathrm{~Gb} / \mathrm{s}$ & $\begin{array}{l}\text { Peer to } \\
\text { peer }\end{array}$ & NRT \\
\hline $\begin{array}{l}\text { CoMP } \\
\text { signaling }\end{array}$ & $774 \mathrm{~Kb} / \mathrm{s}$ & $\begin{array}{l}\text { Peer to } \\
\text { peer }\end{array}$ & RT \\
\hline $\begin{array}{l}\text { Handover } \\
\text { inter-ring }\end{array}$ & $\begin{array}{l}0.1 \mathrm{~Gb} / \mathrm{s} \text { up } \\
\text { and down }\end{array}$ & Centralized & NRT \\
\hline $\begin{array}{l}\text { Handover } \\
\text { intra-ring }\end{array}$ & $0.1 \mathrm{~Gb} / \mathrm{s}$ & $\begin{array}{l}\text { Peer to } \\
\text { peer }\end{array}$ & NRT \\
\hline
\end{tabular}

while NRT (or best effort) traffic consists of (some) data traffic, data for the CoMP mechanism, and handover $(\mathrm{HO})$ signaling. The most strict latency requirement comes from CoMP signaling, which should experience less than $1 \mathrm{~ms}$ end-to-end latency. Given that such traffic may traverse one node in the backbone network to ensure communication between 2 eNodeBs in two different mobile backhaul networks (here, rings), the per-ring maximum latency for RT traffic is thus at most $500 \mu \mathrm{s}$. This paper quantifies the impact of CoS management during slot formation on traffic latency. We first describe how slots are formed in a non-CoS managed network. 


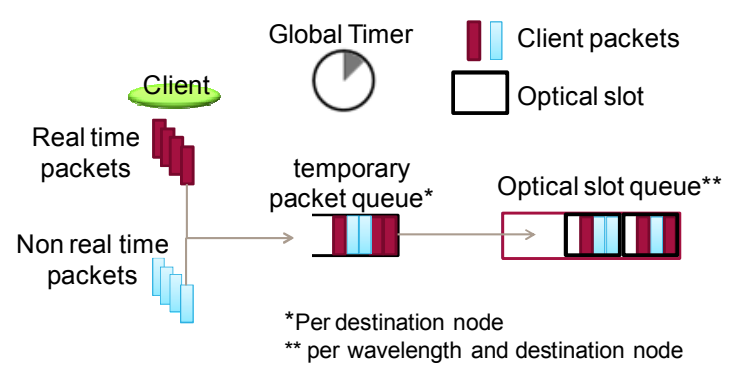

Figure 2: Optical slot formation without CoS management.
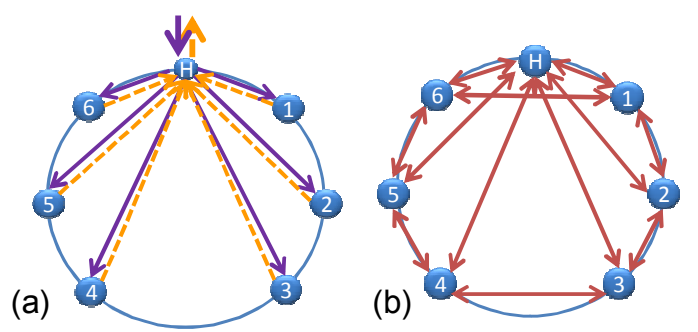

Figure 4: Traffic pattern: (a) centralized; (b) peer to peer.

\section{Slot formation without CoS management}

Without CoS management, client packets with any CoS are mixed in the same optical slot, as described in Fig. 2. At a POADM (eNodeB) node, packets arriving from clients (e.g. mobile terminals) are placed in a temporary packet queue identified by the destination (eNodeB) node. Once enough packets have arrived, the temporary queue is emptied and the optical slot is formed and placed in the optical slot queue, waiting for its insertion on the channel. To cap optical slot formation time, we use a timer, which is triggered by the arrival of the first client packet, and whose expiration causes the completion of the formation of the optical slot.

Slot formation with CoS management

As described in Fig. 3, temporary queues are now identified not only by destination node but also per CoS, such that each optical slot contains packets from the same CoS. The utilization of per-CoS queues enables sending RT traffic before NRT traffic. CoS differentiation is performed during slot assembly only; due to transparency, all slots are treated equivalently by the transport layer, irrelevant of their CoS.

\section{Scenario description}

We consider a POADM ring connecting 7 eNodeBs; each base-station is connected to a POADM node. As described in Fig. 4 the traffic sent between base-stations, belonging to the same ring, remains inside the ring. The traffic exchanged with base-stations on other rings or with the Internet (via the backbone) is transmitted via a selected OSS node, which we call "hub" and denote by $\mathrm{H}$.

As mentioned earlier we consider 6 types of traffic. Each type of traffic has a specific pattern: centralized traffic is between any node

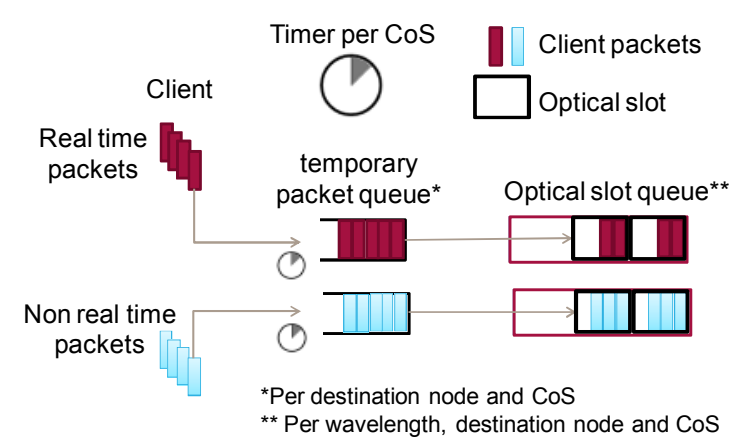

Figure 3: Optical slot formation with CoS management.

and the backbone (i.e., it transits via $\mathrm{H}$ ) while peer-to-peer traffic is between any two eNodeBs. The pattern and per-flow demands for each type are summarized in Tab. 1 .

The dimensioning of the POADM ring that ensures the support of such traffic demand is computed using algorithm ${ }^{4}$ and results in a 6 -wavelength ring with 3 transponders on the hub and 1 transponder on every other node.

\section{Performance evaluation}

First we evaluate the performance of the POADM solution with no CoS management in the 7-node mobile backhaul network. The slot duration is set to $8 \mu \mathrm{s}$ and client traffic is assumed to be Poisson. A load of 1 corresponds to the demand of Tab. 1 and other loads are generated by scaling this reference load. Propagation (a few $\mu$ s in a 7-node mobile backhaul ring) and insertion/extraction on the medium (1 slot duration each) are small compared with the target real-time traffic latency $(500 \mu \mathrm{s})$ such that queuing delays, which are reported here, are representative of the latency experienced by client data within the ring.

We first set the (global) timer to $500 \mu \mathrm{s}$; Fig. 5 shows the mean queuing delay for each traffic type. Seemingly counter-intuitively, the queuing delay decreases as load increases for low loads; this is because queuing delay is then dominated by the time to actually fill the optical slots $^{5}$. At higher loads the queuing delay mainly depends on channel congestion and queuing delay increases, as expected. Fig. 5 shows that the mean queuing delay of all traffic classes is well below the timer limit for load up to 0.88 . The $\sim 10 \%$ wasted load is due to the impossibility to fully fill slots with client data. This problem can be solved by allowing segmentation of client packets in several slots; this is out of the scope of this work. Note that there is no loss up to load 0.85 and we measured a client frame loss below $1 \mathrm{e}-3$ up to load 0.9 .

In Fig. 6 we show the impact of CoS management. We consider that half of the data traffic is RT and the other half is NRT. We keep 


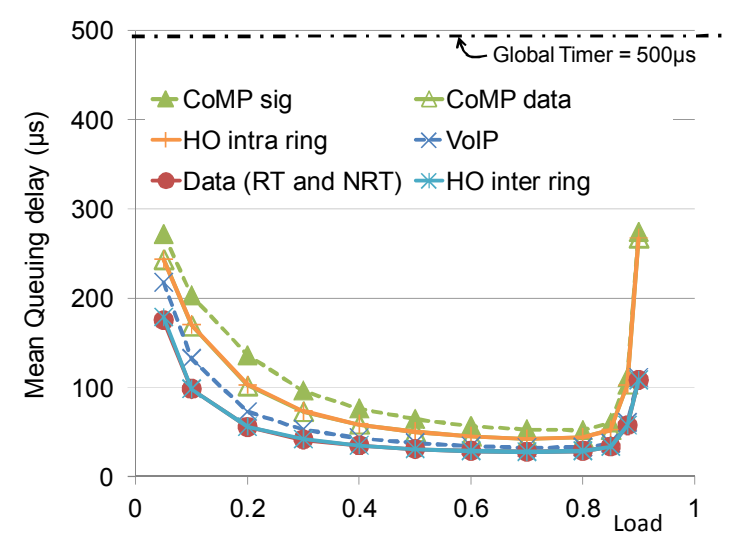

Figure 5: Mean queuing delay without CoS management.

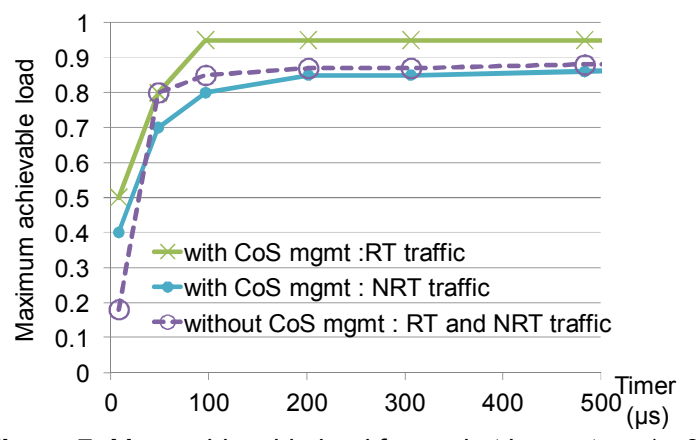

Figure 7: Max. achievable load for packet loss rate $<1 \mathrm{e}-3$. a timer of $500 \mu \mathrm{s}$ for RT traffic and set a timer of $1 \mathrm{~ms}$ for NRT traffic. Both RT and NRT queuing delays are higher than in the no-CoS management scenario. The queuing delays remain below the respective timer values. However, as we can see in Fig. 6, the CoS management policy gives an advantage to RT traffic, since we can achieve a load close to 1 for a packet loss probability below $1 \mathrm{e}-3$. This is at the expense of a lower maximum amount of NRT traffic that can be carried in the network for a given client frame loss rate. Indeed in Fig. 6; NRT traffic can still achieve a load of 0.85 but beyond this value the queuing delay is higher with than without CoS management: RT traffic experiences almost no loss while NRT traffic experiences high losses. Figs. 5 and 6 , show that, whichever CoS management policy is used, the proposed optical slot switching ring fulfills the strict latency constraints of LTE advanced mobile backhaul network.

Fig. 7 shows the impact of the timer (no CoS management: global timer; CoS management: RT timer) on the maximum achievable load for each traffic class. Without CoS management we can reach a load of 0.8 while ensuring a queuing delay for any traffic of $50 \mu \mathrm{s}$. Using CoS management we can still achieve a load close to 1 with a timer of $100 \mu \mathrm{s}$ and load $>0.8$ using a timer of $50 \mu \mathrm{s}$. For very short timers $(<200 \mu \mathrm{s})$ the maximum load achieved for NRT traffic is impacted by the prioritization of RT

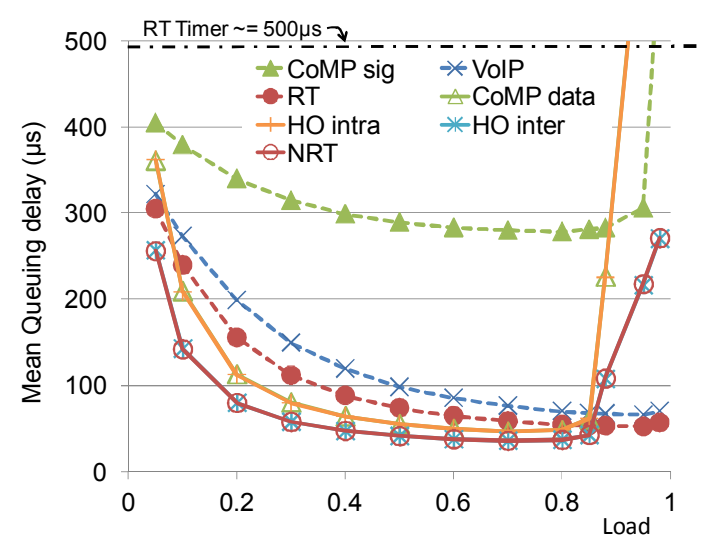

Figure 6: Mean queuing delay with CoS management.

traffic but remains $>0.7$ for a timer of $50 \mu \mathrm{s}$, and $>0.8$ for a timer of $100 \mu \mathrm{s}$.

\section{Conclusions}

We showed that an optical slot switching network fulfills the latency constraints of a next generation LTE advanced switching mobile backhaul network, which can reach as low as a few hundreds of microsecond in the considered segment. Even without differentiated CoS handling, real-time traffic can be supported with load up of to $88 \%$. When CoS handling is used while forming slots it is possible to support even more real-time traffic, at the expense of a reduced non-real-time maximum carried load. Without CoS management, we showed that we can ensure a queuing delay of $50 \mu$ s while reaching a load of $80 \%$ for both RT and NRT traffic; with CoS management we can ensure a queuing delay of $100 \mu$ s while reaching a load of nearly $100 \%$ for RT and $80 \%$ for NRT traffic.

\section{Acknowledgements}

Work supported in part by CELTIC+ SASER project.

\section{References}

[1] Y. Pointurier et al., "Dimensioning and energy efficiency of multi-rate metro networks," IEEE/OSA Journal of Lightwave Technology, vol. 30, no. 22, Nov. 2012.

[2] Q. Wei et al., "Multicast in Mobile Backhaul with Optical Packet Ring," in Proc. STWiMob, Oct. 2013.

[3] D. Chiaroni et al., "Packet OADMs for the next generation of ring networks," Bell Labs Technical Journal, vol. 14, no 4, pp. 263-285, Winter 2010.

[4] B. Uš umli $\square$ et al., "Optimal dimensioning of the WDM unidirectional ECOFRAME optical packet ring," Springer Photonic Network Comm., vol. 22, no. 3, Jul. 2011

[5] N. Benzaoui et al., "Electronic architectures of optical slot switching nodes," in Proc. ONDM, April 2013.

[6] ] S, Brueck, "Backhaul Requirements for Centralized and Distributed Cooperation Techniques", ITG Heildleberg, July 2010.

[7] NGMN alliance, "Guidelines for LTE Backhaul Traffic Estimation," White paper, 2011. 
